\title{
Erratum to "Additive Schwarz domain decomposition methods for elliptic problems on unstructured meshes"
}

\author{
Tony F. Chan ${ }^{\text {a) }}$ and Jun Zou ${ }^{\mathrm{a}, \mathrm{b})}$ \\ a) Department of Mathematics, University of California at Los Angeles, Los Angeles, \\ CA 90024-1555, USA \\ E-mail: chan@math.ucla.edu \\ ${ }^{b)}$ Computing Center, Chinese Academy of Sciences, Beijing 100080, PR China \\ Received 8 February 1995 \\ Communicated by C. Brezinski
}

The following are corrections which were inadvertently not incorporated into the final published version of our paper [1]:

Page 336, line 20: " $O_{i}^{h}=\operatorname{supp} \phi_{i}^{H}$ " should be " $O_{i}^{H}=\operatorname{supp} \phi_{i}^{H}$ ";

Page 340, line -7: "(26)" should be "(8)";

Page 344, lines 3 and 8: "arbitrarily shaped" should be "shape regular";

Page 344, line 15: "maximum" should be "minimum";

Page 344, line 18: " $1 \leq \delta_{i} \leq p$ " should be " $1 \leq i \leq p$ ".

\section{Reference}

[1] T.F. Chan and J. Zou, Additive Schwarz domain decomposition methods for elliptic problems on unstructured meshes, Numer. Algor. 8 (1994) 329-346. 